\title{
APROXIMACIÓN EMPÍRICA A LA INTERACCIÓN DE VERBOS Y ESQUEMAS CONSTRUCCIONALES, EJEMPLIFICADA CON LOS VERBOS DE PERCEPCIÓN
}

\author{
José Ma García-Miguel \\ Universidad de Vigo \\ gallego@uvigo.es
}

\begin{abstract}
Resumen
Para el estudio de la interacción entre léxico verbal y sintaxis se propone una aproximación basada en corpus, utilizando los datos provisionales del proyecto ADESSE. EI examen de los datos muestra la alta flexibilidad sintáctica de cualquier verbo, pero también cómo cada verbo o cada clase semántica de verbos tiende a organizarse siguiendo un conjunto más o menos limitado de patrones sintácticos más frecuentes. Tomando como ejemplo las principales variaciones construccionales de los verbos de percepción del español, se muestra cómo los esquemas sintácticos más frecuentes con los verbos de percepción representan asociaciones semánticamente coherentes entre el significado del verbo y el significado del esquema, cómo los esquemas alternantes proporcionan conceptualizaciones alternativas de situaciones similares, y cómo los verbos más frecuentes con un esquema sintáctico sirven de modelo o punto de atracción para la producción o interpretación de construcciones similares con otros verbos.

PALABRAS CLAVE: estructura argumental, esquemas sintáctico-semánticos, verbos de percepción.
\end{abstract}

\begin{abstract}
In this paper, we take a corpus-based approach to verbs and argument structure, using data from the ADESSE project. The observation of real data shows the high syntactic flexibility of almost any verb, but it also shows that each verb or each semantic class of verbs tends to adopt a more or less limited set of frequent syntactic patterns. Using as examples the main constructional alternations with Spanish perception verbs, it is shown how the most frequent syntactic constructions with perception verbs represent a semantically coherent integration of verb meaning and construction meaning, how constructional alternations provide alternate conceptualizations of similar scenes, and how the most frequent verbs within a syntactic schema act as a model or as an attractor for the production and interpretation of similar constructions with other verbs.
\end{abstract}

KEY WORDS: argument structure constructions, perception verbs.

\section{Introducción}

\subsection{Verbos y esquemas}

Las interacciones entre léxico (verbos) y gramática (sintaxis) han constituido y siguen constituyendo un problema básico de la teoría lingüística contemporánea. Predicados (verbos) y argumentos de predicados (nominales) son fundamentales en la representación del mundo por medio del lenguaje y en el desarrollo de la interacción lingüística. La aproximación que se seguirá en este trabajo concibe el lenguaje básicamente como un sistema de signos, esto es, de asociaciones entre estructuras fónicas significantes y estructuras 
conceptuales relativas al mundo como significados. Una segunda dimensión del lenguaje especialmente relevante para lo que sigue tiene que ver con los procesos de categorización y esquematización, que en las últimas décadas han sido puestos de relieve por la Lingüística Cognitiva: para la comprensión del mundo y del lenguaje entendemos los casos particulares como ejemplares o instancias de categorías más generales, más abstractas o esquemáticas, $o$ encontramos parecidos de familia entre diferentes instancias de uso del lenguaje. Por último, este trabajo pretende seguir una aproximación basada en el uso, no sólo porque se adhiere a la idea funcionalista clásica de que las lenguas son instrumentos de interacción social, sino también porque en la línea de Hopper (1987) o los estudios recogidos por Barlow y Kemmer (2000) entiendo que tanto la significación como las categorías y estructuras gramaticales 'emergen' de experiencias previas de uso contextualizado del lenguaje. Un corolario de esta aproximación es que la frecuencia de uso es un factor fundamental en la conformación de la estructura lingüística.

Los principios anteriores se aplican igualmente a las estructuras gramaticales. La unidad básica es la construcción, entendida también como signo, asociación convencional de significante y significado. Como en Gramática de Construcciones (Goldberg, 1995; Kay y Fillmore, 1999; Croft, 2001), entiendo que los esquemas sintácticos son inherentes a las expresiones concretas que los realizan, y que en el proceso de esquematización no hay límites claros entre el dominio del léxico y el de la sintaxis, sino un continuo en el que intervienen diferentes grados y caminos de esquematización. Tomemos un ejemplo real, sacado de una obra de teatro de Antonio Gala

\section{(1) Como corra mucho, no le vemos el pelo [HOT: 22, 35]}

Sin dificultades, podemos reconocer ahí un caso particular de la construcción prefabricada no ver el pelo, o más exactamente 'no verle el pelo <alguien $>_{\mathrm{s}}<\mathrm{a}$ alguien $>_{1}$ ", donde hemos hecho abstracción de la selección temporal y de la especificidad de los participantes. Esa construcción, a su vez, es un caso particular de la construcción más esquemática '<alguien $>_{\mathrm{S}}$ ver $<$ algo $>_{\mathrm{D}}<\mathrm{a}$ alguien $>_{1}$, , que por un lado es sólo una de las realizaciones

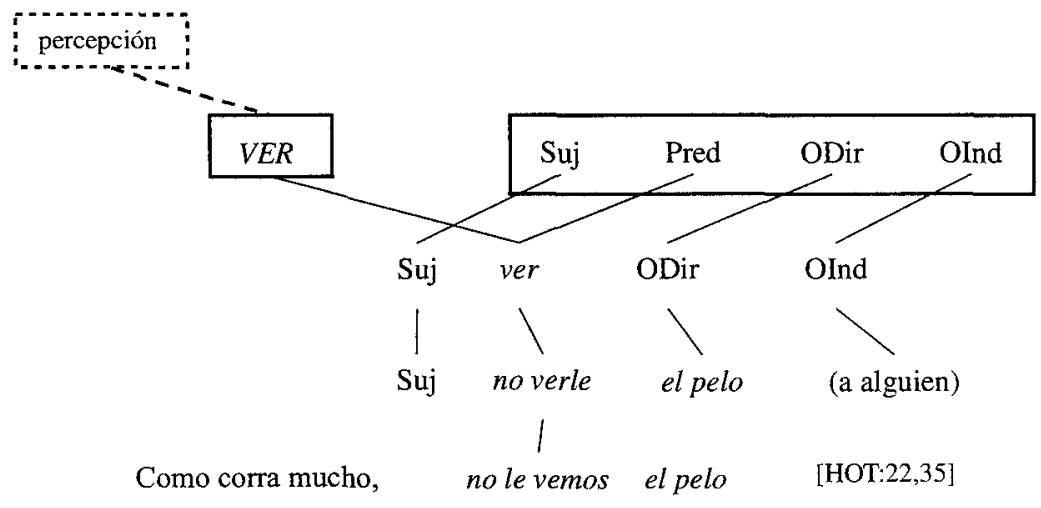

Figura 1

1 "No verle el pelo a alguien. No verle desde hace mucho tiempo" (M. Moliner. D.U.E., s.v. pelo). 
sintácticas posibles del verbo ver, y por otro lado, es un caso particular del esquema sintáctico $<$ Suj Pred ObjDir ObjInd $>$. Siguiendo con el proceso de abstracción, ver es en el léxico del español uno de los verbos de percepción visual, y el esquema ditransitivo es uno de los esquemas triactanciales. La Figura 1 ilustra gráficamente este proceso de esquematización y categorización.

Ante fenómenos como éstos, la mayoría de los lingüistas suelen formular el problema en términos de qué elementos léxicos caben en una estructura sintáctica y en qué estructuras sintácticas cabe un verbo dado. En mi opinión, un desarrollo adecuado de ese problema puede formularse en términos de uso registrado y en términos de procesos de categorización y esquematización. Debemos preguntarnos por las motivaciones del uso de un verbo dado en un esquema sintáctico dado (por ejemplo, la relación entre el uso de un verbo como ver y el uso de un esquema sintáctico como $<$ Suj - Pred - ObjDir - ObjInd $>$ ). En sentido inverso, la cuestión es qué relación hay entre una expresión como verle el pelo a alguien y otros usos de un esquema como el ditransitivo, o qué relación hay entre esa misma expresión y otros usos del verbo ver. Como queda dicho, la motivación de tales relaciones debe buscarse y comprobarse en el uso real. Y para ello nada mejor que un corpus textual, junto con el correspondiente análisis sintáctico y semántico. Expondré a continuación las principales características de la base de datos ADESSE, diseñada específicamente para el estudio de la interrelación entre verbos y construcciones.

\subsection{La base de datos ADESSE}

El proyecto ADESSE (Alternancias de Diátesis y Esquemas Sintáctico-Semánticos del Español $)^{2}$ tiene como objetivo conseguir una base de datos con información sintácticosemántica sobre los verbos del español. Tal base de datos no es sino una ampliación de la Base de Datos Sintácticos del Español Actual (BDS), que contiene el análisis sintáctico ("manual") de las casi 159.000 cláusulas del corpus ARTHUS, de 1,5 millones de palabras, con textos de diferentes géneros - narrativa $(37 \%)$, oral $(19 \%)$, ensayo (17\%), teatro (15\%), periódicos (12\%) - procedentes de España (79\%) y América $(21 \%)$. En la BDS se registran las construcciones de 3436 verbos diferentes [lemas], anotando para cada cláusula del corpus rasgos generales de la cláusula, esquema sintáctico y para cada argumento sintáctico nuclear los rasgos siguientes, si procede: función sintáctica, categoría sintáctica, concordancia verbal / clítico pronominal, animación, determinación, número y preposición. A toda esa información sintáctica de la BDS, en ADESSE estamos añadiendo información semántica relativa a sentidos verbales, clases de verbos y roles semánticos. De este modo, una cláusula del corpus como la ejemplificada en (1) recibe en la base de datos de ADESSE los rasgos recogidos en la Tabla 1 , entre otros.

2 E] proyecto ADESSE (http:/webs.uvigo.es/adesse/) está siendo financiado por el Ministerio de Ciencia y Tecnologia (BFF2002-01197 y HUM2005-01573) y por la XUNTA de Galicia (PGIDIT03PXIC30201PN), y contó con una ayuda inicial de la Universidad de Vigo. Además del firmante de este texto, también forman parte del equipo investigador Francisco Albertuz, Susana Comesaña, Lourdes Costas, Susana Martínez, Gael Dos Santos y Antonio Rifón. Todas los recuentos que se ofrecen en este texto deben tomarse con cautela, pues la base datos de ADESSE está en construcción y sólo pueden obtenerse por ahora datos provisionales. 
Tabla 1. Principales rasgos semánticos y sintácticos de un registro en ADESSE

\begin{tabular}{|l|l|l|l|}
\hline Texto: & \multicolumn{2}{l|}{ Como corra mucho, no le vemos el pelo [HOT:22,35] } \\
\hline Verbo: & VER \\
\hline Sentido: & 1 “Percibir por medio de la vista" \\
\hline Clase verbal: & \multicolumn{2}{l}{ Mental - percepción } \\
\hline Figurado: & Sí & \\
\hline & Estructura sintáctico-semántica \\
\hline Rol semántico: & A1 (Perceptor) & A2 (Percepción) & A3 (Poseedor A2) \\
\hline Función Sintáctica: & Sujeto & ObjDir & ObjInd \\
\hline Animación: & Humano & Concreto & Humano \\
\hline Concordancia / Clítico: & $1^{\text {a plural }}$ & -- & le (masc) \\
\hline Unidad sintáctica: & -- & FN & -- \\
\hline
\end{tabular}

Junto con los registros de cláusulas individuales, disponemos en la base de datos también de presentaciones resumidas concernientes a verbos, clases de verbos, esquemas, etc. lo cual nos permite obtener información global acerca de entre otras cosas esquemas sintácticosemánticos de verbos del español y su frecuencia de uso, verbos que ocurren en cierto esquema construccional, esquemas de los verbos pertenecientes a una clase semántica, clases semánticas de los verbos registrados en un esquema sintáctico, o cualquier otra correlación entre rasgos anotados en la base de datos. Por ejemplo, la Figura 2 es una muestra parcial de los datos generales relativos al verbo ver, donde consta una definición, su clasificación semántica, los roles de participantes registrados en el corpus, los esquemas sintácticosemánticos más frecuentes en voz activa, junto con su frecuencia, en los que se indica la asociación entre función sintáctica, representada mediante una letra ( $, D, I, O$, etc.) y rol semántico, representado mediante un número.

Un somero examen de los datos nos muestra que la mayoría de los verbos no poseen un único significado, sino un conjunto abierto de sentidos relacionados de los que muchas veces podría, eso sí, abstraerse un significado abstracto flexible y adaptable a diferentes contextos de uso. De la misma manera, los datos niegan una determinación unívoca de la estructura sintáctica por parte del léxico y muestran que casi todos los verbos pueden construirse en más de un esquema sintáctico. De los esquemas sintácticos admitidos por un verbo, unos son más típicos, más usuales que otros. Tampoco las clases semánticas de verbos determinan univocamente las posibilidades sintácticas. Entre dos verbos semánticamente similares puede haber diferencias en cuanto a qué esquemas sintácticos admite cada uno y qué esquemas de los admitidos son relativamente más típicos en cada caso. Todo esto no es óbice para que podamos encontrar tendencias y motivaciones relativos a la integración de significado léxico y significado construccional en la interacción de verbos y esquemas sintácticos. 


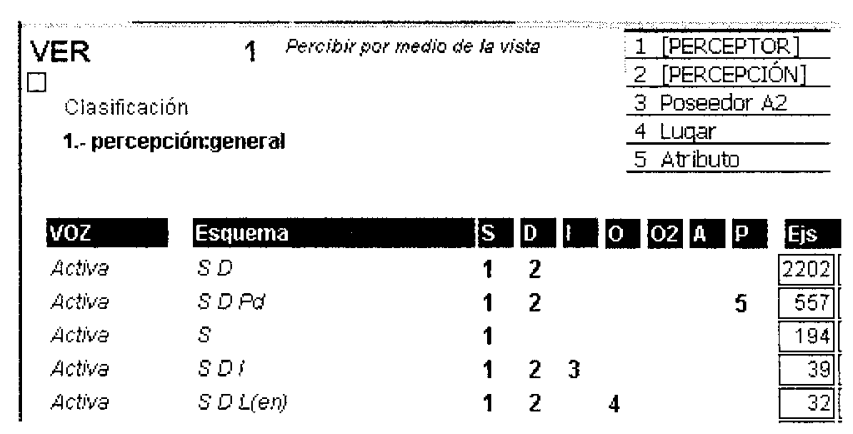

Figura 2. Parte de la ficha del verbo ver en ADESSE.

\section{Eventos, participantes y clases de eventos}

Los verbos y los esquemas sintácticos de una lengua nos proporcionan instrumentos para representar escenas o situaciones del mundo real o imaginado. Al utilizar una palabra (un verbo) o una construcción gramatical (esquema) estamos conceptualizando una situación como realización o ejemplar de cierta clase o tipo. El significado del verbo evoca una estructura conceptual compleja en la que se integra también el conocimiento enciclopédico sobre situaciones a las que es aplicable un lexema y sobre las condiciones pragmáticas de uso de cada unidad lingüística. Las situaciones designadas se diferencian por el modo de interrelacionarse las entidades que intervienen en ellas. Tales entidades son los participantes o actantes recogidos en las fórmulas esquemáticas que dan cuenta de la valencia o estructura argumental de un verbo. Por ejemplo:

(2) a. COMER: alguien (A1) come algo (A2)

b. CASARSE: alguien (A1) se casa con alguien (A2)

c. SENTIR: alguien (A1) siente algo (A2)

d. REGALAR: alguien (A1) regala algo (A2) a alguien (A3)

e. AVISAR: alguien (A1) avisa [de] algo (A2) a alguien (A3)

f. IR: $\quad$ alguien (A1) va de un sitio (A2) a otro (A3) por algún lugar (A4)

Entiendo además que los verbos pertenecientes al mismo grupo semántico comparten en gran medida elementos de su base conceptual, incluyendo el modo de relacionarse los participantes en las situaciones designadas. Por ejemplo, podemos caracterizar esquemáticamente los eventos de percepción como la relación entre un perceptor y un objeto percibido. Esta caracterización esquemática se aplica a nuestra conceptualización de ciertos tipos de situaciones, y forma parte del significado de los verbos de percepción por el hecho de ser verbos de percepción.

Admitiendo como un hecho que cada situación es única, pero que el léxico verbal de las lenguas nos permite abstraer lo que tienen de similar situaciones parcialmente diferentes, en el proyecto ADESSE estamos acometiendo la clasificación de los verbos del español en un inventario de clases conceptuales o tipos de eventos. La Tabla 2 muestra los dos primeros niveles ("macroclase" y "clase") de la clasificación jerárquica utilizada. 
Tabla 2. Clases verbales en ADESSE

\begin{tabular}{|l|l|r|l|}
\hline MACROCLASE & CLASE & VERBOS & Ejemplos \\
\hline \multirow{4}{*}{1 MENTAL } & 11 Sensación & 196 & Gustar \\
\cline { 2 - 5 } & 12 Percepción & 80 & Ver \\
\cline { 2 - 5 } & 13 Cognición & 91 & Saber \\
\hline \multirow{3}{*}{ 2 RELACIÓN } & 21 Atribución & 118 & Ser \\
\cline { 2 - 5 } & 22 Posesión & 92 & Tener \\
\hline \multirow{4}{*}{ 'PROCESOS MATERIALES' } & 31 Espacio & 410 & Ir \\
\cline { 2 - 5 } & 32 Cambio & 439 & Abrir \\
\cline { 2 - 5 } & 33 Otros hechos & 183 & Coger \\
\cline { 2 - 5 } & 34 Comportamiento & 153 & Reir \\
\hline 4 COMUNICACIÓN & 260 & Decir \\
\hline 5 EXISTENCIA & 89 & Haber \\
\hline 6 CAUSATIVOS Y DISPOSITIVOS & 65 & Hacer \\
\hline TOTAL VERBOS CLASIFICADOS & 2176 & \\
\hline
\end{tabular}

La mayoría de las clases se dividen a su vez en subclases (por ejemplo: cognición-conocimiento y cognición-creencia) hasta completar en este punto un total de 54 opciones. Una exposición más detallada de los criterios de clasificación utilizados en ADESSE, incluyendo una comparación con otros sistemas de clasificación de verbos puede verse en Albertuz (en prensa).

En ADESSE, cada clase conceptual se asocia con una serie de papeles típicos del dominio cognitivo descrito. Por ejemplo:

(3) Sensación: $\quad<$ Experimentador, Estimulo>

Percepción: $\quad<$ Perceptor, Percibido $>$

Cognición: $\quad<$ Conocedor, Contenido $>$

Posesión: $\quad<$ Poseedor, Posesión>

Posesión-transferencia: <Poseedor-inicial, Posesión, Poseedor-final>

Espacio-desplazamiento: <Móvil, Origen, Destino, Trayecto $>$

Espacio-localización: <Móvil, Lugar>

Comunicación: <Emisor, Mensaje, Receptor>

Son muchas las teorías lingüísticas que hacen referencia a alguna lista básica de papeles o roles semánticos generales (agente, paciente, instrumento, etc), aunque no hay ni parece que vaya a haber alguna vez ninguna lista definitiva y la aproximación basada en listas de roles ha sido sometida discusión (cf. Levin y Rappaport Hovav, 2005: 38-44). Al fin y al cabo, los papeles semánticos no son sino una interpretación de nuestro conocimiento sobre las situaciones. En último extremo, cada situación particular y, en cierto nivel de abstracción, cada verbo particular "defines a distinct set of participant roles that reflect its own unique 
semantic properties (e.g. the subject of bite is a slightly different kind of agent from the subject of chew)." (Langacker, 1991: 284) ${ }^{3}$. Sin embargo, siguiendo los procesos comunes de categorización, podemos establecer generalizaciones que abarquen diferentes eventos y diferentes verbos. De acuerdo con lo sugerido más arriba en la Figura 1, hay al menos dos vías básicas de abstracción o categorización de eventos, una basada en el léxico y otra basada en las estructuras sintácticas. La primera está ligada a la estructura jerárquica del vocabulario y a su agrupación en campos semánticos y dominios cognitivos. Es la que nos permite agrupar los verbos en clases y asociar éstas con una lista de roles semánticos que no son sino generalizaciones sobre verbos particulares ${ }^{4}$. La otra vía de abstracción nos lleva a los esquemas sintácticos, los cuales obligan a una caracterización máximamente esquemática de los eventos, pues un mismo esquema puede usarse con verbos de muy diferente significado y relativos a muy diferentes dominios cognitivos. Algunas de las más solventes propuestas de generalización apelan a nociones aspectuales, combinadas con protoroles o macroroles (Dowty, 1991; Van Valin y LaPolla, 1997), o al concepto de cadena causal combinado con el de perfil (Langacker, 1991; Croft, 1991 y 1998). Sin pretender discutir en este artículo tales propuestas, apelaré más bien al uso efectivo que hacemos de verbos y de construcciones y a cuáles son las realizaciones sintácticas normales o frecuentes para un verbo o grupo de verbos, y a cuáles son los verbos y clases de verbos más normales o frecuentes con ciertos esquemas sintácticos. En último término, de lo que se trata es de ver cómo compatibilizar el significado de verbos y construcciones y de acomodar el significado de unos y otras cuando se generaliza el uso de una combinación aparentemente incoherente. Intentaremos ejemplificar todo ello con las más evidentes características semánticas y sintácticas de los verbos de percepción del español.

\section{Verbos de percepción en ADESSE y sus construcciones}

Aceptaremos la definición propuesta por Enghels y Roegiest, según la cual "la percepción se define como un tipo particular de proceso cognitivo por la que un individuo (animado, generalmente humano) experimenta un estímulo exterior y obtiene por este estímulo información sobre el mundo exterior" (Enghels y Roegiest, 2004: 47). Esta definición nos indica que deben esperarse dos participantes inherentes a todo proceso perceptivo: el indivi-

3 También Van Valin y LaPolla utilizan ocasionalmente etiquetas específicas para cada clase de verbos, aunque no les reconocen estatus teórico alguno: "they are merely mnemonics for argument positions in logical structure $\langle\ldots\rangle$ these labels are useful in that they indicate the subclass of a predicate; hence coGNizER means 'first argument of a two-place state predicate of cognition', JUDGMENT means 'second argument of a two-place state predicate of propositional attitude"' (Van Valin y LaPolla, 1997:128).

4 La idea de que las lenguas poseen una red de sentidos verbales relacionados y que los roles de participantes son específicos de cada tipo de eventos está siendo aplicada también en el proyecto FrameNet (Fillmore et al, 2003; Subirats, 2004). En FrameNet cada 'frame' o marco conceptual define sus propios 'frame elements' o argumentos semánticos de modo que, por ejemplo, los argumentos semánticos asociados con el verbo tell (Speaker, Addressee, Message) son muy diferentes de los asociados con el verbo throw (Agent, Theme, Source, Path, Goal). Por otro lado, tanto la perspectiva de FrameNet como la nuestra se sitúan conscientemente en lo que Moreno Cabrera (2003:18-27) llama cosmomorfismo semántico, en contraposición al gramatomorfismo semántico. Precisamente, lo que queremos investigar en ADESSE es cómo la conceptualización del mundo elaborada por el léxico interactúa con el significado aportado por las estructuras gramaticales. 
duo que experimenta un estímulo exterior (perceptor) y el estímulo experimentado (entidad percibida o percepto).

En la base de datos de ADESSE, la clase de procesos de percepción incluye provisionalmente en el momento de redactar estas páginas 84 verbos, registrados en aproximadamente 11.000 cláusulas. Lo mismo que en cualquier otra de las clases semánticas previstas en ADESSE, se trata de una lista abierta que incluye los verbos semánticamente próximos a los más típicos. En este sentido nuestra aproximación es similar a la propuesta por Goldberg (1995: 133-136) al concebir las clases de verbos como "similarity clusters", lo cual es coherente con un modelo conexionista de la memoria y el conocimiento. Con ese criterio y esa matización se incluyen verbos de percepción visual (ver, mirar), percepción auditiva (oír, escuchar), otros tipos de percepción sensorial (oler, saborear, tentar), así como verbos de percepción general (advertir, notar, percibir, detectar, percatarse,...). Lo que proporciona mayor diversidad léxica a la clase es la existencia de un amplio grupo de verbos relativos a maneras de ver (contemplar, observar, atisbar, avistar, avizorar, divisar, entrever, escrutar, escudriñar, ojear, otear, presenciar, visionar, vislumbrar; examinar, espiar, explorar, fijarse, inspeccionar, registrar, remirar, revisar, vigilar,...). En la periferia semántica de la clase de percepción situamos los verbos de búsqueda y hallazgo (buscar, encontrar, hallar, localizar) y los causativos de percepción, que son también verbos de transmisión de conocimientos o información (mostrar, enseñar, exhibir, descubrir, presentar, revelar, ocultar, camuflar; manifestar, exponer, exteriorizar, insinuar). La Tabla 3 recoge las frecuencias de los verbos de percepción más usuales, por eso mismo aquellos que mejor caracterizan la clase y que sirven de plantilla conceptual para otros verbos de percepción.

Tabla 3. Verbos de percepción más frecuentes en $\mathrm{ADESSE}^{5}$

\begin{tabular}{|l|c|c|}
\hline \multicolumn{1}{|c|}{ VERBO } & Registros & Esquemas \\
\hline ver & 3486 & 33 \\
\hline mirar & 1282 & 36 \\
\hline oír & 927 & 17 \\
\hline encontrar & 843 & 21 \\
\hline buscar & 619 & 8 \\
\hline escuchar & 436 & 14 \\
\hline mostrar & 314 & 12 \\
\hline descubrir & 290 & 13 \\
\hline contemplar & 233 & 8 \\
\hline observar & 212 & 7 \\
\hline
\end{tabular}

5 No se han incluido en esa lista dos verbos relativamente frecuentes en los que la percepción no es el dominio cognitivo más prominente, aunque está siempre comprendida en el significado del verbo: leer (535 registros) y presentar ( 363 registros). Tampoco está incluido el verbo sentir (1128 registros), que es más general y abarca tanto lecturas de percepción como de sensación. 
En la Tabla 4 se muestran los esquemas sintáctico-semánticos más frecuentes con los verbos de percepción, entendiendo cada esquema como una correspondencia específica entre funciones sintácticas y roles semánticos

Tabla 4. Esquemas construccionales más frecuentes con verbos de percepción

\begin{tabular}{|l|l|l|l|l|r|r|r|}
\hline \multicolumn{1}{|c|}{ VOZ } & Esquema & Sujeto & ObjDir & ObjInd & Otros & Cláusulas & Verbos \\
\hline Activa & S D & Perceptor & Percibido & & & 6802 & 57 \\
\hline Activa & S D Pd & Perceptor & Percibido & & Atributo & 643 & 8 \\
\hline Activa & S & Perceptor & & & & 581 & 26 \\
\hline Activa & S D & Iniciador & Percibido & & & 466 & 15 \\
\hline CP media & S Ps & Percibido & & & Atributo & 213 & 4 \\
\hline Activa & S D I & Iniciador & Percibido & Perceptor & & 188 & 9 \\
\hline CP refl. & S d & Perceptor & Percibido & & & 160 & 13 \\
\hline Activa & S L(hacia) & Perceptor & & & Orientación & 78 & 1 \\
\hline & Otros & & & & & 2222 & \\
\hline TOTAL & & & & & & 11353 & 86 \\
\hline
\end{tabular}

Lo que comprobamos, tanto si observamos cada verbo individualmente, como si observamos globalmente el grupo de verbos de percepción es que casi siempre es posible un amplio repertorio de esquemas sintáctico-semánticos para un verbo o un grupo de verbos. Los hechos ponen en cuestión la idea de que el léxico determine univocamente las estructuras sintácticas. Incluso ponen en cuestión la idea de que un verbo o una clase de verbos se asocie unívocamente con una lista cerrada de argumentos semánticos inherentes. En cambio, sí debemos admitir que la distribución de un verbo no es aleatoria y que el significado verbal determina el contexto de uso y está determinado por él. En consecuencia, entendemos que la estructura argumental de un verbo o de una clase de verbos no es sino una generalización a partir del uso registrado. Por eso, en vez de participantes obligatorios o inherentes a un sentido verbal o a una escena, nuestros recuerdos del uso lingüístico nos proporcionan ciertas expectativas o probabilidades sobre la referencia a un tipo de participante en la escena denotada por el verbo o la clase verbal, esto es, la lista de roles argumentales para un verbo dado debería incluir la frecuencia de cada rol y no limitarse a una lista más o menos estricta. Lo mismo puede decirse de la asociación entre (clases de) verbos y esquemas sintácticos. Veremos a continuación como se producen variaciones a partir de las asociaciones no marcadas.

\section{Transitividad}

Hemos visto en la Tabla 4 que el esquema sintáctico transitivo es, con diferencia, el más frecuente con verbos de percepción, aunque caben muchas otras alternativas construccionales. La motivación de que un (grupo de) verbo(s) aparezca frecuentemente en un determinado esquema sintáctico debe buscarse en el significado y en el uso. El significado de un 
esquema sintáctico se establece (y se aprende) como generalización a partir del significado de las expresiones particulares que instancian o ejemplifican tal esquema y para que la combinación sea coherente, el significado del verbo (y de los participantes en las situaciones designadas por él) debe poder conceptualizarse como una instancia, un caso particular, del significado del esquema (cf. Goldberg, 1995: 50). Empíricamente, el valor de una construcción se comprueba observando con qué verbos se combina y con qué significado se asocia frecuentemente. La Tabla 5 muestra qué clases semánticas de verbos son más frecuentes en el esquema transitivo en español.

Tabla 5. Clases verbales más frecuentes en el esquema transitivo $<\mathrm{SUJ}+\mathrm{ObjDir}>$ en ADESSE

\begin{tabular}{|l|c|c|}
\hline CLASE <argumentos> & $\begin{array}{c}\text { Cláusulas } \\
\text { transitivas }\end{array}$ & $\begin{array}{c}\text { Verbos en } \\
\text { cláusulas transitivas }\end{array}$ \\
\hline Cognición <Conocedor, Contenido> & 8663 & 68 \\
\hline Percepción <Perceptor, Percibido> & 6802 & 57 \\
\hline Posesión <Poseedor, Posesión> & 5660 & 9 \\
\hline Comunicación <Emisor, Mensaje> & 3134 & 122 \\
\hline Actividad <Agente, Actividad> & 2999 & 21 \\
\hline Modificación <Agente, Afectado> & 2892 & 381 \\
\hline Sensación <Experimentador, Estímulo> & 2299 & 32 \\
\hline Desplazamiento <Iniciador, Móvil> & 1512 & 69 \\
\hline
\end{tabular}

De la tabla se deduce que no parece haber, al menos en español, un único 'prototipo' de cláusula transitiva; pero si atendemos a los casos más frecuentes, parece concebirse siempre algún tipo de relación asimétrica entre dos participantes que se orienta desde el Sujeto hacia el Objeto ${ }^{6}$ :

- Un Agente que actúa sobre un Paciente

- Un Experimentador de actividad mental que dirige su atención hacia ciertas Experiencias o ciertas ideas

- Un Poseedor que mantiene el control sobre alguna Posesión

Es pertinente señalar que la Tabla 5 muestra cierta discrepancia en la distribución estadística de las clases semánticas en términos de frecuencia de ejemplares ['token frequency'] frente a frecuencia de tipos ['type frequency']: en los textos parece haber más cláusulas de

\footnotetext{
6 La definición de la transitividad como relación asimétrica está inspirada en Langacker (1991:309-313). Es común tradicionalmente, y más aún desde Hopper y Thompson (1980), tomar como prototipo de transitividad las cláusulas de acción en las que un agente afecta físicamente a un paciente. Vázquez Rozas (2004), sin embargo, argumenta que es más básica la causalidad intencional que la física. Otra vía de generalización apela a las propiedades aspectuales, y en concreto a la telicidad (que de algún modo implica la culminación de un cambio de estado). El problema es que esta última no es aplicable a estados como los de posesión y los denotados por la mayoría de los verbos de percepción, aunque Moreno Cabrera (2003:161-2) interpreta los verbos de cognición y percepción como procesos adherentes, esto es, como cambios en las relaciones que una entidad mantiene con otras.
} 
procesos mentales y relacionales, pero en el léxico parece haber más verbos de acción y modificación. Algunos estudios como los de Bybee y Thompson (1997) atribuyen mayor relevancia a la frecuencia de tipos (número de verbos) que a la frecuencia de ejemplares (número de cláusulas). En mi opinión, ambos criterios de distribución contribuyen a conformar la función prototípica de la transitividad y refuerzan la idea de que no hay un único prototipo.

Por otro lado, también en lo que concierne al uso del esquema transitivo encontramos diferencias entre unos verbos de percepción y otros, que son causa y consecuencia de sus diferencias de significado. Enghels y Roegiest (2004) han mostrado que las diferencias cognitivas y fisicas entre la percepción visual (ver) y la percepción auditiva (oir) son correlativas del mayor uso con esta última de la preposición a y del clítico le, entre otras diferencias sintácticas.

Otra dimensión de variación ampliamente reconocida y estudiada (por ejemplo, Rodríguez Espiñeira, 2000) es la existente entre percepción física directa y percepción mental indirecta. La primera concierne a personas, objetos concretos y eventos, como en (4) y (5). La segunda concierne primariamente a la percepción de ideas y se expresa típicamente mediante cláusulas finitas, como en (6).

(4) a. Déjame ver el novio [JOV:94,34]

b. Te asomas a la ventana y ves toda la sierra [MAD:361,13]

(5) a. Alli veíamos pasar las cofradias [SEV:208,11]

b. Me asomé y vi que el coche seguía parado junto a la acera [LAB:115,12]

(6) Martino vio que yo plantaría cara a los Cantanottes [SON:261,20]

La extensión (metafórica) desde la percepción sensorial hasta la percepción mental parece una posibilidad 'natural' de los verbos de percepción y se registra con la mayoría de ellos. Pero está también determinada por el uso registrado y muestra claramente 'efectos ligados a la frecuencia'. Aunque debe considerarse sólo como indicio indirecto del tipo de percepción que se asocia a cada verbo, ver es frecuente en objeto clausal, mientras que mirar es muy poco frecuente, aunque no imposible.

Tabla 6. Participante percibido en la construcción transitiva con VER y MIRAR

\begin{tabular}{|l|c|c|}
\hline & VER & MIRAR \\
\hline Humano & $32 \%$ & $65 \%$ \\
\hline No humano & $42 \%$ & $30 \%$ \\
\hline Clausal & $26 \%$ & $5 \%$ \\
\hline
\end{tabular}

Con todo, la construcción transitiva es simplemente la forma canónica de representar las situaciones que implican percepción. Todos los verbos de percepción admiten construcciones o interpretaciones alternativas al esquema transitivo. Nos preguntaremos en los próximos apartados qué es lo que permite, en cada caso, asociaciones alternativas entre verbo y esquema. 


\section{Alternativas construccionales}

No muy lejos del esquema conceptual básico que relaciona un perceptor y un objeto percibido se sitúan algunas construcciones sintácticas que no se adecuan al esquema transitivo y que presentan conceptualizaciones alternativas de situaciones relacionadas con la percepción.

Por ejemplo, verbos como oler permiten expresar percepción olfativa mediante el esquema conceptual típico de los verbos de percepción, con perceptor como sujeto y objeto percibido como complemento directo

(7) Es claro que tenemos suerte de que nos haya tocado oír el animal y no olerlo [HIS: 161,8$]$

O alternativamente, pueden construirse con sujeto y complemento preposicional. En este caso cabe un dativo para referirse al perceptor del olor.

(8) Me hueles a esa caza violenta, el vello de tus brazos me trae un relente de bosque y de sangre [ZOR:35,27]

Hay una clara diferencia de conceptualización entre las estructuras de (7) y de (8), pues mientras la primera está orientada desde el perceptor, que debe expresarse obligatoriamente, la segunda se centra en la apariencia de una entidad, lo que hace casi obligatorio el complemento preposicional que describe esa apariencia, y completamente secundaria la expresión del perceptor (sólo 2 ejemplos en nuestra base de datos sobre un total de 54 ejemplos con esta variante). No obstante, las lenguas podrían llegar a generalizar una u otra alternativa construccional y el español, como muchas otras lenguas, generaliza la perspectiva que se orienta desde el perceptor.

Otro caso destacable de alternancia sintáctica y conceptual lo tenemos en la afinidad que muestra el verbo mirar entre la percepción visual y la orientación espacial. Esto justifica el que mirar se construya frecuentemente con complementos locativos, especialmente locativos direccionales que alternan con el complemento directo, como en (9), aunque también locativos que expresan trayectoria u origen como en (10), además de la posibilidad de expresar la localización del evento o de cualquiera de los participantes en él.

(9) a. Mira hacia la ventana, coge una piedrecita y la tira contra ella [1IN:34,24]

b. La chica miró a la ventana, luego a él y, finalmente, se encogió de hombros [MIR:44,23]

c. Mira la ventana, ya no se ve el cielo más que sacando la cabeza. [SON:320,22]

(10) a. Miréporlaventana con los prismáticosy vi una señora lavando los platos [LAB:113,29] b. Miraba desde la ventana, apoyando la sien derecha en el cristal. [MIR:123, 1]

Hanegreefs (en prensa) ha analizado los complementos preposicionales locativos con mirar y distingue entre: (i) los locativos dinámicos que al mismo tiempo denotan el objeto percibido (= complemento verbal de régimen preposicional); (ii) los dinámicos que indican la trayectoria de la mirada (= complemento verbal de régimen preposicional o adjunto) y (iii) los estáticos que sitúan al perceptor o al objeto percibido (= adjunto). La motivación 
de tales complementos locativos, y especialmente, la posibilidad de que los complementos direccionales denoten el objeto percibido se entenderá mejor a partir de la Figura 3, que desarrolla una similar de Hanegreefs.

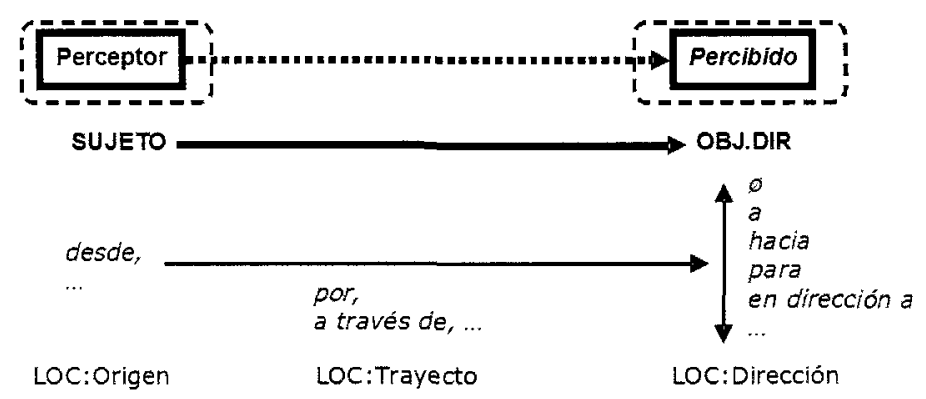

Figura 3. Percepción y complementos locativos con mirar

Existe una homología entre la orientación inherente a la construcción transitiva, la orientación de las relaciones de percepción visual derivada de la configuración física del ser humano y la direccionalidad de las relaciones espaciales. De este modo, el objeto percibido es también el objeto hacia el que se orienta la mirada y la codificación como orientación permite inferir la existencia de percepción, y viceversa. Formalmente, hay una continuidad entre la expresión como objeto directo, y la expresión como direccional, pudiendo quedar los complementos con $a$ como los de (9) indeterminados en cuanto a su estatus bien como complemento direccional o bien como objeto directo preposicional, salvo que un clítico acusativo lo codifique explícitamente como objeto directo (lo miró a él).

\section{Elementos adicionales}

Hemos visto que los procesos de percepción, por su propia naturaleza, incluyen dos participantes inherentes, perceptor y percibido, que suelen corresponderse con sujeto y objeto directo del esquema transitivo, aunque caben conceptualizaciones y esquemas sintácticos alternativos. También cabe la posibilidad de utilizar esquemas sintácticos con más de dos constituyentes dependiendo del predicado. Esto puede ocurrir sin necesidad de que existan más de dos participantes en la situación designada, simplemente otorgando mayor o menor prominencia a diferentes partes o facetas de la entidad percibida.

\subsection{Predicaciones secundarias}

En las construcciones con predicativo, incluyendo las construcciones de infinitivo y construcciones con locativo, existe un desdoblamiento sintáctico y semántico de lo percibido. Muchos estudios de orientación generativista ven las construcciones de (11a) como estructuras de verbo más "cláusula reducida" o "cláusula mínima" (por ejemplo, Demonte y Masullo, 1999: 2501), equivalentes sintáctica y semánticamente a las construcciones verbo más cláusula completiva de (11b) 
(11) a. Ver a Pedro al fondo de la sala / sentado / sentarse / tocando el piano b. Ver que Pedro está el fondo de la sala / que está sentado / que se sienta / que Pedro está tocando el piano

Rodríguez Espiñeira ha estudiado y destacado las diferencias entre estas dos construcciones. Está en primer lugar el hecho de que la construcción con completiva se asocia más fácilmente a la percepción indirecta o mental, como hemos señalado más arriba. Además, la construcción con predicativo tiene tras el verbo no uno, sino dos constituyentes sintácticos lo que implica una estructura semántica específica: en la construcción con predicativo la percepción es "bifocal, no proyecta globalmente un acontecimiento, sino que permite enfocar simultáneamente dos aspectos relevantes de un suceso: el participante involucrado y el evento en que interviene" (Rodríguez Espiñeira, 2000: 81). Siguiendo una línea similar de argumentación, entiendo que es aplicable aquí el análisis que elabora Langacker (1995) para lo que se ha tratado a veces como estructuras de "elevación": las construcciones con predicativo en general y las de infinitivo en particular otorgan prominencia al sujeto "lógico" del predicativo asignándole la función de objeto directo y perfilando la relación entre sujeto y objeto. Eso no quiere decir que sólo sea relevante la relación directa entre sujeto y objeto, pues es cierto, como señalan Demonte y Masullo (1999: 2505), que notar a alguien cansado no implica lógicamente notar a alguien. La relación entre sujeto y objeto está mediatizada por el predicativo. Siguiendo a Langacker, el objeto directo está metonímicamente por el predicado secundario y actúa como punto de referencia con respecto a él. Es decir, la escena se conceptualiza indicando que el perceptor establece contacto mental con una entidad y a través de ella accede mentalmente al proceso o estado representado por el predicativo.

\subsection{Prominencia parte-todo}

Las diferencias de prominencia parte-todo derivan del hecho de que la percepción de una parte implica la percepción (parcial) del todo, que se convierte así en participante inherente de la situación, típicamente expresado en forma de dativo posesivo. De ahí las alternancias del tipo de las mostradas en (12), que otorgan diferentes grados de prominencia a la parte o al todo, según qué es lo que se conceptualice en cada caso como entidad relevante (como participante central, en el sentido de García-Miguel, 1995:27-52):

(12) a. Ver a alguien / ver su cara / verle la cara

b. Oír al niño / oír la voz del niño ${ }^{7}$

c. A ver cuándo me llamas nonno $\langle\ldots>$; El día que te lo oiga me darás la vida! [SON:282,8]

El hecho de que el verbo mirar perfile también la orientación del perceptor y admita por tanto complementos direccionales nos ofrece más alternativas construccionales si queremos mencionar tanto persona como parte del cuerpo. Además de la parte del cuerpo como objeto y el poseedor bien como posesivo (13a) bien como dativo (13b), también podemos tener un 
complemento direccional (13c) o seleccionar la persona como objeto directo y especificar la parte del cuerpo como complemento direccional o locativo (13d-e)

(13) a. - No mires mis ojos- dijo él en un tono imperioso [DIE:150,34]

b. Cuando uno sacrifica una res no se atreve a mirarle los ojos [CRO:56,28]

c. Blanca mira a los ojos de Guillermo [COA:49,3]

d. Carmina le tomó la mano y le miró a los ojos [TER:112,7]

e. Robert empezó a apartarse mirándola en los ojos [GLE:155,14]

Esta última construcción es rara con otros verbos de percepción, salvo en aquellas variantes dialectales que difuminan la oposición semántica entre ver y mirar, como en el ejemplo siguiente de la mexicana Elena Poniatowska:

(14) Ella también comia viéndolo a la cara [DIE:63,20]

En todo caso, tanto este apartado como el anterior nos muestran que el número de argumentos de una predicación no es algo que derive sin más del número de referentes presentes en la situación evocada. Es algo que el hablante construye conceptualmente al elegir una construcción sintáctica $u$ otra. Y esa elaboración conceptual puede hacerse refiriéndose a diferentes partes o diferentes facetas de lo que desde otro punto de vista podría se una sola entidad.

\subsection{Participantes adicionales: Verbos Causativos de Percepción}

Las estructuras con tres actantes sintácticos con verbos de percepción no son exclusivas de aquellas construcciones en que lo percibido se desdobla semánticamente de alguna manera. También es posible expresar participantes adicionales abriendo la ventana de atención (en el sentido de Talmy, 1996) hasta abarcar el iniciador de la cadena causal que posibilita el proceso perceptivo. Como con algunas otras clases verbales, la causatividad da lugar a unidades léxicas específicas (mostrar, enseñar, exhibir, ...) con significados que elaboran el sentido "dejar/hacer ver" y estructuras sintácticas triactanciales, típicamente ditransitivas, aunque la expresión de alguno de los tres participantes inherentes a estos verbos puede ser opcional

(15) Victoria Guzmán le mostró el cuchillo ensangrentado [CRO:16,28]

Advertir y descubrir abarcan sentidos causativos y no causativos, de modo que además de la estructura triactancial causativa (16) permiten diferentes valores para la estructura transitiva, bien perceptor más objeto percibido (17), bien iniciador o desencadenante más objeto percibido o mostrado (18)

(16) ¿Dónde se había visto que una chica decente le descubriera a un hombre un local? [US:89,5]

(17) Al abrir los ojos, descubrió la figura inmensa de un hombre que le observaba [TER:10,17] 
(18) Aquel acontecimiento fue como la iluminación que descubre un espacio insospechado [MIR:53]

De todas maneras, lo que me parece más interesante de los verbos causativos de percepción es comprobar cómo influye su esquema sintáctico habitual (Suj-ODir-OInd) en el sentido que adoptan. En principio, el esquema es simplemente una necesidad derivada de la inclusión de un participante más (el iniciador o causante) que debe añadirse a los esenciales en los procesos perceptivos (el perceptor y lo percibido). Pero lo que encontramos típicamente en el esquema Suj-ODir-OInd son verbos de transferencia de posesión y de comunicación, tal como muestra la Tabla 7.

Tabla 7. Clases verbales más frecuentes en el esquema $<$ Suj ODir OInd $>$ en ADESSE

\begin{tabular}{|l|c|c|}
\hline Clase & Cláusulas & Verbos \\
\hline Comunicación & 2363 & 90 \\
\hline Posesión: transferencia & 2192 & 46 \\
\hline Actividad genérica & 544 & 9 \\
\hline Espacio: desplazamiento & 438 & 40 \\
\hline Percepción & 376 & 28 \\
\hline
\end{tabular}

Varios trabajos han insistido en que la adopción de una construcción está motivada no por un significado abstracto atribuible al esquema sino por el significado específico de los verbos típicos con esa construcción. En particular, tanto Kemmer y Verhagen (1994) como Fauconnier y Turner (1996) defienden que los verbos de transferencia pueden servir de modelo para las construcciones causativas tanto analíticas como sintéticas. Pero lo que encontramos con los causativos de percepción del español va más allá del simple hecho de que la causatividad se modele sintáctica y semánticamente como la transferencia. Es que la polisemia de la mayoría de los verbos causativos de percepción incluye junto al significado básico "mostrar, dejar ver" como en (19a) y (20a), también sentidos aún más propiamente de transferencia, bien transferencia de conocimientos o habilidades (19a) bien transferencia de información (20b).

(19) a. Le estaba enseñando las fotos de Carmela [CAI:92,12]

b. Le enseñaron artimañas de comadronas [CRO:42,2]

(20) a. Manifiesta cariño y simpatía por los animales [PAI:172,15]

b. La idea me entusiasmó y así se lo manifesté [SUR:61,25]

El uso frecuente de manifestar, exponer, insinuar y otros verbos semejantes como expresión de proceso verbal es especialmente significativo de cómo interactúan significado verbal y significado construccional, afectando el uso habitual de este último al valor que adquiere al primero al combinarse ambos. 


\section{Omisión de participantes inherentes}

Hasta ahora hemos utilizado el supuesto de que todo proceso perceptivo, y por tanto todo verbo de percepción, implica dos participantes inherentes y hemos considerado solamente construcciones en las que al menos hay un perceptor y un objeto percibido, con construcciones alternativas si focalizamos diferentes facetas de los percibido o si añadimos participantes adicionales. Pero también es posible encontrar verbos de percepción que no explicitan los dos participantes considerados inherentes. Veamos qué ocurre si con un verbo de percepción no mencionamos el perceptor o lo percibido.

\subsection{Omisión del participante percibido}

Si utilizamos un verbo de percepción en un esquema sintáctico monoactancial, no hay lugar para al menos uno de los participantes inherentes. El resultado normal es que si lo percibido no es mencionado, resulta evocado con carácter genérico, como posibilidad, o como inferencia contextual:

(21) a. Bebía y miraba, miraba y bebía [CAR: 113,3]

b. El primo divagaba. David escuchaba en silencio [JOV: 131,23]

c. El que busca, encuentra [HIS: 165, 2]

d. ¿Dónde vas a estar mejor que aquí? - Ya encontraré. [HOM: 56, 22]

Resulta necesario distinguir aquí entre significado 'codificado' (un solo participante como sujeto) y significado 'evocado', dependiendo éste último enteramente del significado del verbo y de estrategias discursivas contextualizadas, sin que exista necesariamente ningún referente que pueda ser considerado como participante percibido. De hecho, muchos de los usos intransitivos que registramos con ver y mirar, oír y algunos otros verbos de percepción debemos considerarlos como marcadores discursivos. Se trata de fórmulas como ya veo, ya ves, ya veremos, ¿ves? ¿viste?, no veas, vamos a ver, mira, oiga, .. que guían el desarrollo del discurso oral y la interacción hablante-oyente:

(22) Esté... El llano en llamas, que hay una parte, ¿viste?, de un ... Hlamado ... yo digo, ese tipo para mí es un genio y vos no ... [BAI:419,30]

(23) Enc.- ¿Qué opinas de los jornales de hoy?

Inf.- $j \mathrm{~J}$ e, je! Desde luego, ¡qué indiscretas son las preguntas! Pues mira...

Enc.- ¿Tú crees que son indiscretas?

Inf.- Verás, por ejemplo, yo, para mí, en estos momentos pues con lo que ganamos, para mí personalmente, me... defiendo. [MAD:197]

(24) - Vamos a ver, Emilia, tus padres, ¿dónde nacieron?

- Pues verás, mi padre nació aquí en Sevilia. Mi madre, no. [SEV:20,21]

Ante ejemplos como estos, en ningún caso diríamos que hay un argumento sintáctico o semántico de realización nula. Pero aunque lo percibido no exista ahí como argumento sintáctico-semántico, el significado léxico de ver evoca un frame de percepción, en el que una persona dirige su atención hacia algo. Este frame evocado lo utilizamos como trasfondo 
interpretativo en el desarrollo del discurso y sirve de indicación al oyente para que preste atención aunque no se le diga explícitamente a qué tiene que prestar atención. Al mismo tiempo, en un proceso de gramaticalización y de transferencia metonimia, sirve al hablante para mostrar distancia interpersonal respecto del oyente (Chodorowska, 2005) y como prefacio de un nuevo movimiento conversacional

\section{2. ¿Percepción sin Perceptor?}

Más difícil que la omisión de lo percibido es la omisión del perceptor. En general, las reducciones de transitividad que afectan a una actante que en el esquema no marcado sería sujeto, se asocian en español con el uso de la construcción pronominal. Ésta adquiere normalmente con los verbos de percepción las variantes reflexiva (25a), recíproca (25b), mediopasiva (26a), o impersonal (26b) de acuerdo con las características generales esperables de las construcciones pronominales:

(25) a. Si usarais el espejo no para contemplaros, sino para veros, ... [RAT:21,14]

b. Llámame, a ver si ya salgo y tal, y nos vemos [MAD:446,27]

(26) a. A veces se ven cosas raras, ¿no crees? [SUR:88,18]

b. No se los vio más. Desaparecieron. [HIS:162,7]

Nótese de todas maneras que, aunque sintácticamente hay un solo argumento explícito, las construcciones reflexivas y recíprocas implican una doble función (perceptor y percibido) por parte del sujeto, y que tanto la medio-pasiva como la impersonal evocan un participante humano, genérico o inferible contextualmente, que se corresponde en este caso con el perceptor. Sin embargo, hay siempre una defocalización del perceptor, que lo lleva a un muy segundo plano hasta el punto de que puede llegar a ser difícil explicitar quien es o podría llegar a serlo. El ejemplo siguiente es formalmente medio-pasivo, pero cual sea el posible perceptor es menos relevante que el hecho de que 'las casas estaban blancas', es decir lo que se afirma primariamente es la existencia de una entidad y un estado, como condición previa para que algo pueda ser percibido

(27) Las casas, en el centro del césped, se veían blancas [DIE:137]

Se inicia así un camino de extensión semántica, basado en la metonimia, que lleva a que verbos incluidos inicialmente entre los de percepción se usen para referirse a situaciones en las que no hay implicado ningún tipo de perceptor. Es el caso de los verbos encontrar $y$ hallar, que admiten usos recíprocos, como en (28) con sujeto humano, y mediopasivos, como en (29) con sujeto inanimado

(28) El héroe y la heroína se encontraron en la montañita de la plaza Roma [HIS:72]

(29) Sobre todo el flamenco que se puede encontrar en cualquier taberna [SEV:70,31]

Pero que tanto con sujeto humano como con sujeto inanimado pueden equivaler simplemente a "estar", sin que se implique ningún tipo de percepción 
(30) a. Sí, otra vez me encuentro solo, no tengo a nadie más que a ti [TER:110,3]

b. El comando terrorista libanés podría encontrarse en España [1VOZ:5,4,1,52]

(31) a. Citroën, cuya planta de producción se encuentra en la Zona Franca de Vigo [2VOZ:58,2,1,3]

b. Se sorprendieron al ver que las grandes puertas se encontraban cerradas [CAR:176]

Algo similar ocurre con el verbo ver, que ya hemos documentado como reflexivo, recíproco y mediopasivo. Pero en (32), tanto con sujeto animado como inanimado, no se puede decir que tengamos sentido reflexivo ni que haya necesariamente implicado un perceptor externo. Al difuminarse la relevancia del perceptor, verse nos aparece como equivalente de 'ser'

(32) a. El equipo gallego se vio perjudicado por las decisiones arbitrales [1 VOZ:50,2,5,1]

b. Mi vida se ha visto amenazada [GLE:50,3]

c. El trasvase del líquido se vio dificultado por el alto nivel de corrosión [3VOZ:16,2,4,29]

En esta construcción verse va casi siempre seguido de participios como obligado, afectado, sorprendido, envuelto, ... El proceso de desemantización resulta como consecuencia de la inserción del verbo en una configuración sintáctica específica <verse + participio>, pero su repetición pone en marcha un proceso de gramaticalización que podría culminar en el uso de verse como mero auxiliar de construcciones pasivas.

\section{Conclusión}

En este recorrido necesariamente incompleto y algo superficial por las construcciones de los verbos de percepción hemos partido de verbos típicamente bivalentes y prototípicamente transitivos, pero el corpus nos ha mostrado un amplio rango de esquemas construccionales posibles para cualquier verbo y para la clase de verbos de percepción en su conjunto. Rechazada la hipótesis de que el léxico determina unívocamente la estructura sintáctica, podemos ver las alternancias construccionales como variaciones a partir de un esquema conceptual básico (perceptor que dirige la atención hacia una entidad percibida), con alternativas debidas a diferencias de prominencia relativa del perceptor o lo percibido, prominencia de parte o del todo, posibilidad de mención de participantes adicionales o posibilidad de relegar al trasfondo algún participante básico. Con ello, podríamos ver el significado global de una expresión como la combinación del significado de los elementos léxicos, en particular el significado del verbo que evoca cierto tipo de escena, más el significado de la construcción, que otorga mayor o menor prominencia a los participantes en la escena evocada.

Pero hemos podido ver que hay algo más que eso. $Y$ es que no es tan sencillo separar el significado del verbo y el significado de las construcciones. Los unidades lingüísticas no existen aisladamente, ocurren contextualizadas y su aparición en contexto es indisociable de su significado. Los verbos siempre nos aparecen en algún esquema y los esquemas clausales siempre nos aparecen con algún verbo. Pero de todas las asociaciones posibles entre 
verbos y esquemas unas están más fijadas en el uso y en la memoria que otras. La Figura 4 ilustra el hecho comprobado en nuestro corpus de que cada verbo de percepción admite más de un esquema sintáctico-semántico pero no hay ningún esquema en el que encontremos cualquiera de los verbos de percepción. Eso sí, el esquema transitivo con el perceptor (A1) como sujeto y lo percibido (A2) como objeto directo es la construcción prototípica de los verbos de percepción, como comprobamos por su mayor frecuencia tanto en número de cláusulas como en número de verbos. Pero las diferencias de significado entre unos verbos y otros se reflejan en el grado en que pueden llegar a asociarse con otros esquemas sintácticos. Por otro lado, los esquemas sintácticos se asocian también en mayor grado con unos verbos y grupos de verbos que con otros, y eso contribuye a fijar su significado. De este modo, la utilización por ejemplo de un verbo como mirar en una construcción con complemento direccional es forzosamente reminiscente de construcciones similares con otros verbos entre los que figuran verbos de desplazamiento o verbos estáticos de orientación. $\mathrm{E}$ igualmente para cualquiera de los esquemas de la Figura 4 o para cualquier otro de los que hemos considerado en este trabajo. El significado de un esquema es en parte independiente de los elementos léxicos que lo ocupan, pero tal significado no se establece en abstracto y fuera de contexto, sino que se establece a partir del uso que hacemos de él y a partir de los elementos léxicos que suelen aparecer en él.

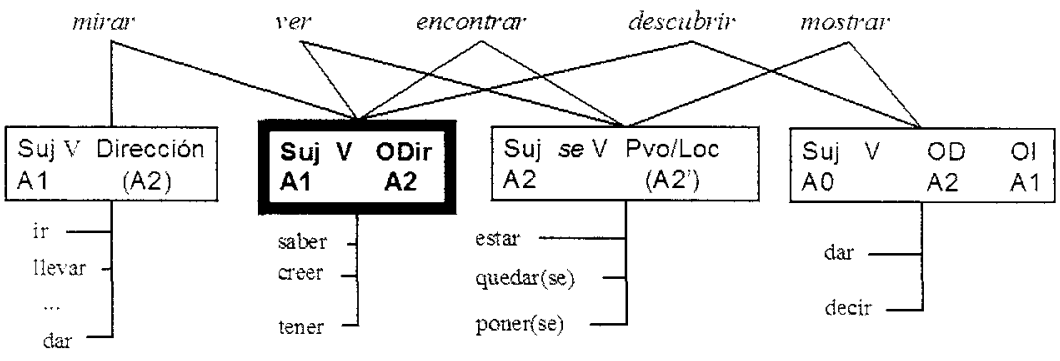

Figura 4

La consecuencia de lo dicho es que el esquema no simplemente matiza, destaca, añade o quita elementos a partir del significado evocado por el verbo. Ni el significado del verbo ni el significado del esquema son constantes y se extienden en un continuo de sentidos que se modulan mutuamente. Es la construcción y la contextualización lo que provoca que encontremos verbos de percepción en expresiones que muy difícilmente podemos considerar de percepción. El caso más claro lo hemos ejemplificado en 7.2 con los verbos encontrarse, hallarse y verse expresando fundamentalmente atribución o localización, o incluso como soporte de pasiva. Pero no es más que un caso particular de un panorama general que ilustramos en la Figura 5. El diagrama muestra parcialmente la red de esquemas sintácticosemánticos usuales con los verbos de percepción, organizada alrededor del esquema transitivo que le sirve de prototipo construccional para la clase. Pero la red se extiende hacia construcciones que son periféricas para la percepción y más centrales para otros dominios conceptuales en los que los verbos correspondientes pasan a incluirse. 


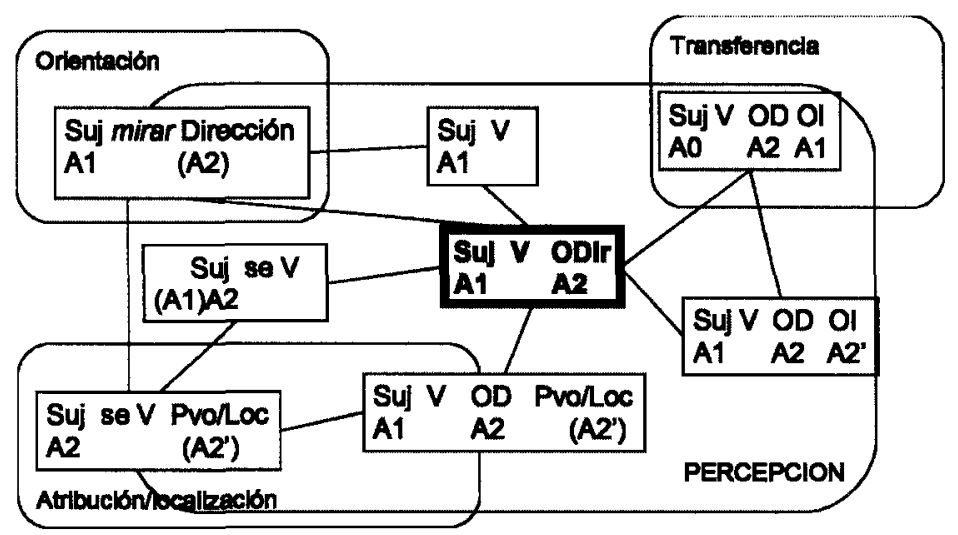

Figura 5

Por supuesto, hay también una base puramente conceptual en la extensión polisémica y en la producción de un significado concreto a partir de las posibilidades que ofrecen los elementos integrados. Pero creo que los datos ofrecidos nos muestran la necesidad de observar el uso real (hablado y escrito) y apuntan a la frecuencia de uso como factor conformador de la lengua y factor de fijación del sentido.

Terminaremos observando que en el uso real y en el aprendizaje las unidades las observamos en contexto; pero a partir de las expresiones reales registradas podemos seguir dos líneas importantes de generalización: una nos lleva por el léxico a descubrir las unidades elementales de organización lingüística del conocimiento del mundo; la otra nos lleva a los esquemas construccionales como patrones convencionales de categorización de situaciones y de modulación de la representación conceptual. Y al recorrer ese camino, es importante mirar alternativamente 'hacia abajo' y 'hacia arriba', esto es, buscar generalizaciones que abarquen todos los datos de naturaleza similar, pero también observando detalles cada vez más minuciosos. Somos conscientes de que muchos de los fenómenos que se han apuntado en estas páginas merecen un análisis mucho más detallado de lo que aquí ha sido posible.

\section{Referencias bibliográficas}

Albertuz, Francisco J. (en prensa): "Sintaxis, semántica y clases de verbos: Clasificación verbal en el proyecto ADESSE". Actas del VI Congreso de Lingüistica General. Santiago de Compostela, 3-7 mayo 2004: http://webs.uvigo.es/adesse/textos/Albertuz-CLG6.pdf.

Barlow, Michael y Suzanne Kemmer (eds) (2000): Usage-based models of language. Stanford, CSLI.

Bybee, Joan y Sandra A. Thompson (1997): "Three frequency effects in syntax", Berkeley Linguistic Society, 23, págs. 65-85.

Croft, William (1991): Syntactic Categories and Grammatical Relations: The Cognitive Organization of Information. Chicago, University of Chicago Press.

Croft, William (1998): "Event Structure in Argument Linking". En Butt, M. y W. Geuder (eds): The Projection of Arguments: Lexical and Compositional Factors. Stanford, CSLI, págs. 21-63.

Croft, William (2001): Radical Construction Grammar: Syntactic Theory in Typological Perspective. Oxford, Oxford University Press. 
Chodorowska, Marianna (2005): "Verás: language in interaction". 11 th Conference on Language, Interaction and Social Organization (LISO), University of California, Santa Barbara, May 12-14th, 2005 .

Demonte, Violeta y Pascual J. Masullo (1999): “La predicación: los complementos predicativos". En Bosque, I. y V. Demonte (dirs ): Gramática descriptiva de la lengua española. Madrid, Espasa, págs. 2461-2523.

Dowty, David (1991): "Thematic Proto-roles and Argument Selection", Language, 67/3, págs. 547619.

Enghels, Renata y Eugeen Roegiest (2004): "Percepción visual y percepción auditiva: La naturaleza del objeto". En Serra, E. y G. Wotjak (eds), págs. 47-59.

Fauconnier, Gilles y Mark Turner (1996): "Blending as a central process of grammar". En Goldberg, A. E. (ed): Conceptual Structure, Discourse and Language. Stanford, CSLI, págs. 113-129.

Fillmore, Charles, Christopher R. Johnson y Miriam R. L. Petruck (2003): "Background to FrameNet", International Journal of Lexicography, 16/3, págs. 235-250.

García-Miguel, José M. (1995): Transitividad y complementación preposicional en español. Santiago de Compostela, Universidade de Santiago.

Goldberg, Adele E. (1995): Constructions: A Construction Grammar Approach to Argument Structure. Chicago / London, University of Chicago Press.

Hanegreefs, Hilde (en prensa): "La construcción preposicional con mirar: un análisis sintáctico-semántico", Boletín de Lingüística, 22.

Hopper, Paul J. (1987): "Emergent Grammar", Berkeley Linguistic Society, 13, págs. 139-137.

Hopper, Paul J. y Sandra A. Thompson (1980): "Transitivity in Grammar and Discourse", Language, 56 , págs. 251-299.

Kay, Paul y Charles J. Fillmore (1999): "Grammatical Constructions and Linguistic Generalizations: the What's X doing Y? Construction", Language, 75, págs. 1-33.

Kemmer, Suzanne y Arie Verhagen (1994): "The Grammar of Causatives and the Conceptual Structure of Events", Cognitive Linguistics, 5/2, págs. 115-156.

Langacker, Ronald W. (1991): Foundations of Cognitive Grammar, Vol. II: Descriptive Application. Stanford, Stanford Univ. Press.

Levin, Beth y Malka Rappaport Hovav (2005): Argument Realization. Cambridge, Cambridge University Press.

Moreno Cabrera, Juan Carlos (2003): Semántica y Gramática. Sucesos, papeles semánticos y relaciones sintácticas. Madrid, Antonio Machado Libros.

Rodríguez Espiñeira, Ma José (2000): "Percepción directa e indirecta en español. Diferencias semánticas y formales", Verba, 27, págs. 33-85.

Serra, Enrique y Gerd Wotjak (eds) (2004): Cognición y percepción lingüústicas. València / Leipzig, Universitat de València/Universität Leipzig.

Subirats Rüggeberg, Carlos (2004): "FrameNet español. Una red semántica de marcos conceptuales". En Serra, E. y G. Wotjak (eds), págs 182-196.

Talmy, Leonard (1996): "The windowing of attention in language". En Shibatani, M. y S. A. Thompson (eds): Grammatical Constructions: Their Form and Meaning. Oxford, Clarendon Press, págs. 235-287.

Van Valin, Robert D. y Randy J. LaPolla (1997): Syntax, Structure, meaning and function. Cambridge, Cambridge University Press.

Vázquez Rozas, Victoria (2004): "Transitividad prototípica y uso", Boletín de Lingüística, 21, págs 92-115. 


\section{Referencias de los textos del corpus citados}

BAI Barrenechea, A. M. (ed.): El habla culta de la ciudad de Buenos Aires. Materiales para su estudio (tomo 2), Buenos Aires, Inst.de Fil. y Lit. hispánicas 'Dr. Amado Alonso', 1987.

CAI Buero Vallejo, A.: Caimán, Madrid, Espàsa-Calpe, 1981.

CAR Colinas, A.: Larga carta a Francesca, Barcelona, Seix Barral, 1986.

COA Fernán Gómez, F.: La coartada, Madrid, Ed. Antonio Machado, 1987.

CRO García Márquez, G.: Crónica de una muerte anunciada, Madrid, Mondadori, 1987.

DIE Poniatowska, E.: Querido Diego, te abraza Quiela y otros cuentos, Madrid, Alianza-Era, 1987.

GLE Cortázar, J.: Queremos tanto a Glenda, Madrid, Alfaguara, 1981, $4^{\text {a }}$ ed.

HIS Bioy Casares, A.: Historias desaforadas, Alianza, Madrid, 1986.

HOM Salom, J.: Un hombre en la puerta, Madrid, Preyson, 1984.

HOT Gala, A.: El hotelito, Madrid, Ed. Antonio Machado, 1988.

JOV Aldecoa, J. R.: Porque éramos jóvenes, Barcelona, Seix Barral, 1986.

LAB Mendoza, E.: El laberinto de las aceitunas, Barcelona, Seix Barral, 1982.

MAD Esqueva, M. y M. Cantarero (eds.): El habla de la ciudad de Madrid. Materiales para su estudio, Madrid, CSIC (Miguel de Cervantes), 1981.

MIR Guelbenzu, J. M.: La mirada, Alianza, Madrid, 1987.

PAI Goytisolo, J.: Paisajes después de la batalla, Barcelona, Montesinos, 1982.

RAT Sánchez Ferlosio, R.: La homilía del ratón, Madrid, Ed. El País, 1986.

SEV Pineda, M. A. de (ed.): Sociolingüística andaluza 2. Material de encuestas para el estudio del habla urbana culta de Sevilla, Sevilla, Universidad de Sevilla, 1983.

SON Sampedro, J. L.: La sonrisa etrusca, Madrid, Alfaguara, 1985.

SUR

TER

García Morales, A.: El sur (seguido de Bene), Barcelona, Anagrama, 1985.

US

Martínez de Pisón, I.: La ternura del dragón, Barcelona, Anagrama, 1988, $3^{\text {a }}$ ed.

Martín Gaite, C.: Usos amorosos de la postguerra española, Barcelona, Anagrama, $8^{\mathrm{a}} \mathrm{ed}$., 1988.

ZOR Nieva, F.: Te quiero, zorra, Madrid, Ed. Antonio Machado, 1989.

IINF Olmo, L. y P. Enciso: Teatro infantil, I, Madrid, Ed. Antonio Machado, 1987.

1VOZ La Voz de Galicia. A Coruña, 30/10/1991.

2VOZ La Voz de Galicia, A Coruña, 22/11/1991.

3VOZ La Voz de Galicia, A Coruña, 23/11/1991. 